\title{
A robotic brain scheme: proposal originated by a modular robotic control
}

doi:10.1080/11762320701455716

\author{
J. Negrete-Martínez \\ Departamento de Biología Celular y Fisiología. IIBm. Unidad Periférica Xalapa, UNAM, and Facultad de Física e \\ Inteligencia Artificial, UV. México
}

\begin{abstract}
A robot brain scheme has been implemented, based on and controlled by analog circuits and micro-controllers. The modules have been classified as: (a) world signal processing, (b) world signal relevance assessing, (c) pre-motor decisions, (d) motor behavior, and (e) planning and sequencing motor behaviors. There are two types of pre-motor processing: main and world signal tracking. Motor behavior includes all the final motion units. Each of these five classes roughly corresponds to areas in a vertebrate's brain and proved to be an effective robotic brain scheme as they assist in the development of greater complexity in robotic brains and a means to compare different implementations. The scheme stresses the importance of motor behavior modules fed by pre-motor decision modules. The pre-motor decision modules aim the movement while the motor behavior module creates the behavior. Finally, the planning and sequencing modules are imperative implementations in a robotic brain.
\end{abstract}

Key words: Robot brain based, brain scheme, pre-motor modules, final motion modules, planning and sequencing modules, arbiter modules.

\section{INTRODUCTION}

The present paper is about a micro-controller brainorganized network - inspired in a previously published architecture (Negrete-Martinez 2006) - that can be mapped into classes corresponding to the gross anatomy and physiology vertebrates' brain areas.

In a previous paper I described a robot control architecture (Negrete-Martinez 2006) focused on self-organization of self-inhibiting modules. The architecture can generate a complex behavior repertoire. The repertoire can be performance-enhanced or increased by modular polyfunctionality and/or by addition of new modules. Every module estimates the distance from a sensor placed in the tool to a beacon. If the distance was smaller than a previously measured distance, the module drives its motor in the same direction of its prior movement; if the distance were larger, the next movement was produced in the opposite direction; but if the movement produces no significant change in distance, the module self-inhibits (a bandit algo-

\footnotetext{
Corresponding Author:

José Negrete-Martínez

Departamento de Biología Celular y Fisiología

IIBm, Unidad Periférica Xalapa, UNAM

and Facultad de Física e Inteligencia Artificial

UV, México Sebastian Camacho \#5, Xalapa, Ver. Mexico, 91000

Tel: +01 2288 1729577, Fax: +012288 172855

Email: jnegrete@uv.mx; jnegretemartinez@yahoo.com
}

rithm version). A self-organization emerges: any module can be the next to take control of the motor activity of the robot once one module self-inhibits. A single module is active at a given time. The modules were implemented as computer procedures and their turn for participation was scheduled by an endless computer program. The overall behavior of the robot corresponds to a reaching attention behavior. This behavior can be easily switched to a running-away attention behavior by changing the sign of the same parameter in each module. Since scheduling a module did not necessarily produce its sustained intervention, the architecture of this 'brain' was actually providing action induction rather than action selection.

\section{THE ROBOT AND CONTROL MODULES}

The robot (a lynxmotion kit) is shown in Figure 1 together with its 'brain' of micro-controllers mounted in a large tablet. The incoming light signal is taken by an IR sensor carried on top of the 'hand' segment of the arm - and processed in a separate tablet. The output of this last tablet feeds the input of the tablet shown in the figure. The movable parts of the robot are the joints, the two front wheels and the mounting for the sensor.

\section{The robot's brain modules and their organization}

Figure 2 depicts the circuit transducing the light signal into voltage (dotted line box) and the nine kinds of micro-controllers implemented for the rest of the brain 


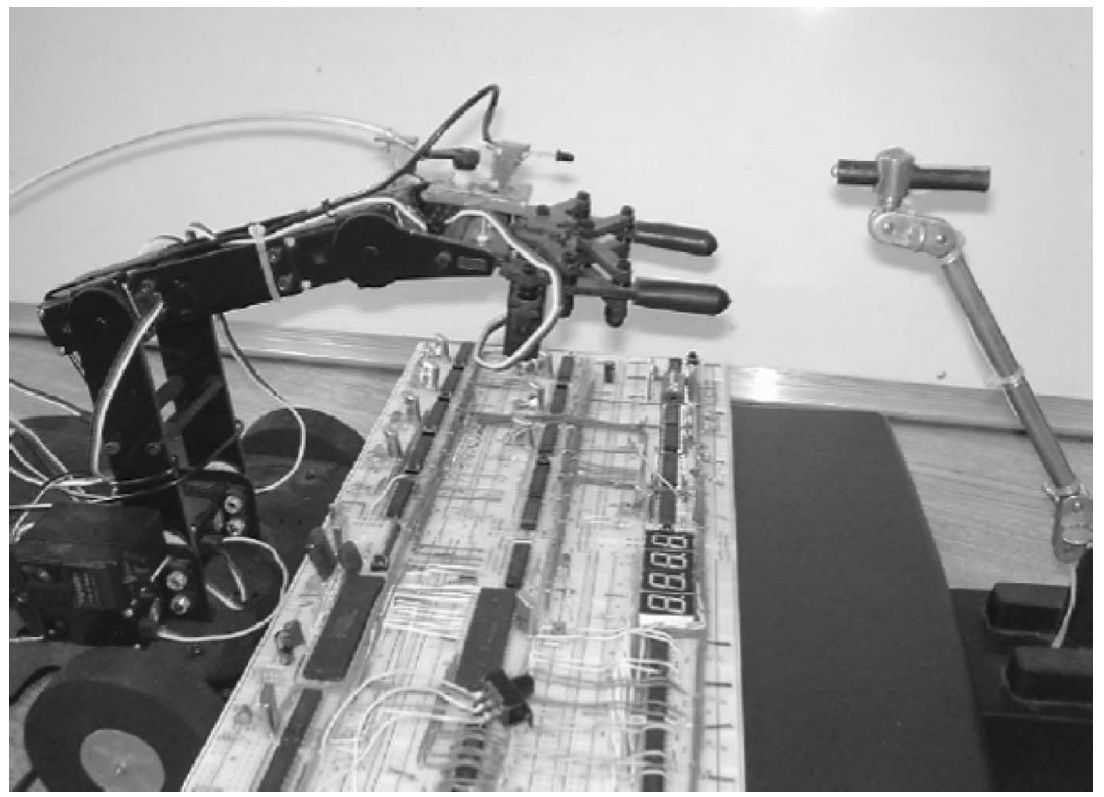

Figure 1 In the center, the tablet with the microcontrollers' brain; to the right, the IR light beacon; and to the left, the mechanical part of the robot with the sensor mounting in the "hand".

(continuous line boxes). The frequency-detector circuit is a gate letting a given flickering frequency signal pass, and transform it into a DC voltage. The light-difference circuit samples this last voltage and stores it into a digital register. This same micro calculates the difference between the previous DC intensity signal and the present one and renders it to a bandit algorithm micro-controller. This micro receives the last-joint-delta-aim sent for a given servo and calculates the next angle increment $(\$ \pm \$$ delta angle) to be taken for the same servo (joint-delta-aim). This increment value is a function of a payoff value and the lastjoint-delta-aim value stored. The payoff value is rendered by a payoff micro-controller (this micro calculates whether the present light intensity difference is significant-positive, significant-negative or irrelevant). The sigma-delta-aim is a micro-controller that adds the angle of the new increment to the present state. The set: joint-delta-aim, lastjoint-delta-aim, sigma-delta-aim, and their corresponding servo are one operating unit called final motion unit (see Figure 2). The scheduling-micro swaps, in a cyclic way, the motion units of the joints. The sensor-scanner and robotaimer are both programmed in one micro-controller. This last micro besides aiming the swinging movement of the mounting (with a modified bandit algorithm) delivers to the wheels-rotation micro the angle that must rotate the platform of the robot in the direction of the position of the light of the beacon. This micro delivers to a final motion unit the time speed and direction each wheel must move given a rotation angle. In Figure 2 the values and signals interchanged among the modules are depicted with solid arrows and the control signals with broken arrows. These last controls are of an on/off nature. Off controls are depicted with round ending arrows. Broken arrows ending at the edge of the final motion units coming from the schedul-

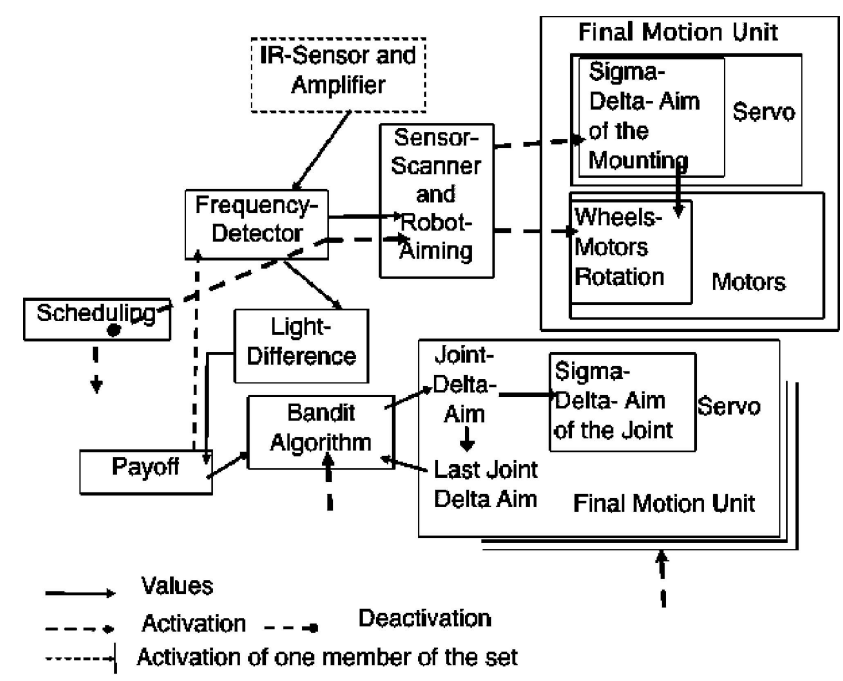

Figure 2 Micro-controllers' network of the robot brain. Final motion unit box with triple edge represent multiple units of the same kind.

ing module indicate that this module activates each one of the motion units (in sequence) for the time the bandit algorithm micro is on. Please note in Figure 2 that the controls coming from the frequency-detector activate only one of two behaviors: the final motion unit of the joints in turn (by default) or the final motion unit of the mounting of the sensor (and its slave final motion unit for the wheels' motors). This switching is produced by activation of the scanner of the mounting and deactivation of the scheduler.

\section{Classification of the modules}

The modules in our robot can be classified as one of four kinds (Figure 3): world signal processing; world signal 


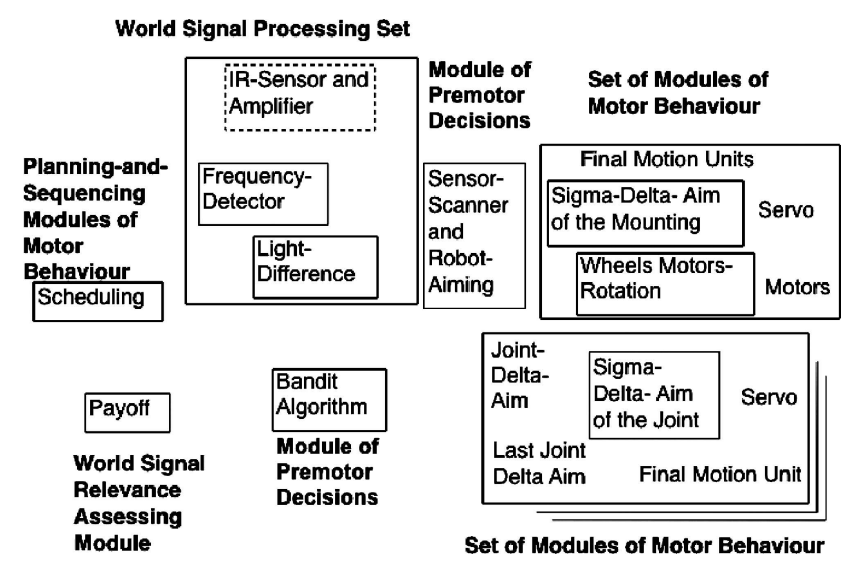

Figure 3 Classification of the robot's modules. The names in bold characters denote the classes.

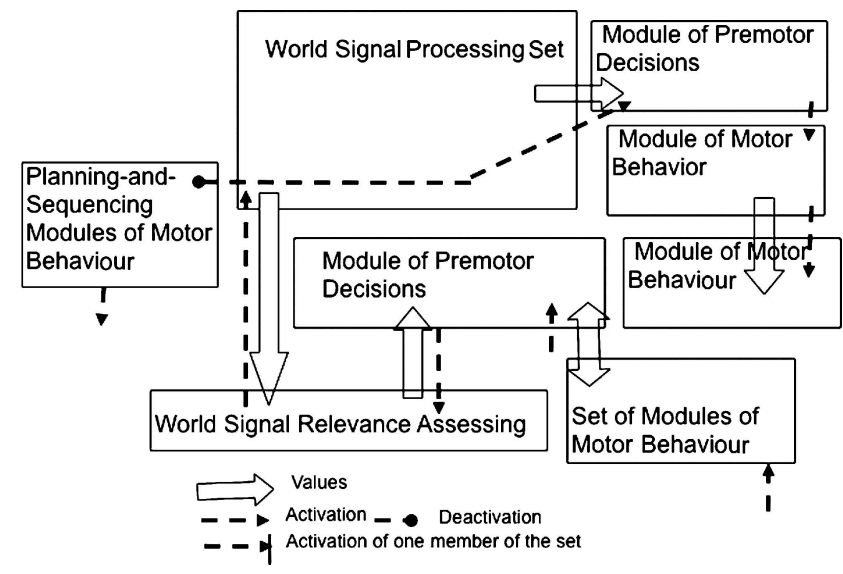

Figure 4 Scheme of a robot brain. This figure is the complete scheme for a robot brain. The classes of Figure 3 are supplemented with the indication of the values and control signals transferred among them.

relevance assessing; pre-motor decisions; motor behavior; and planning-and-sequencing motor behavior.

The world signal processing includes the IR-sensor and amplifier, the frequency-detector and the light-difference circuit. The pre-motor decision includes two modules, the main pre-motor unit and the tracking pre-motor unit; and the motor behavior includes the sigma-delta-aim of the mounting, the wheels motor-rotation and the set of motor behavior for the movement of the arm. All the motor behavior modules include their corresponding motors.

\section{The resulting brain scheme}

In Figure 4 I have added to Figure 3 the direction of the values and the direction of the control signals passed between the classes. The control signals and values supplement the scheme. The generic modular topology is the scheme and the kind of control and the control precedence of one subscheme with respect to others is implementation.

The world-signal processing set sub-scheme corresponds to at least one of the following systems:

visual; auditory, gustatory; and olfactory systems.

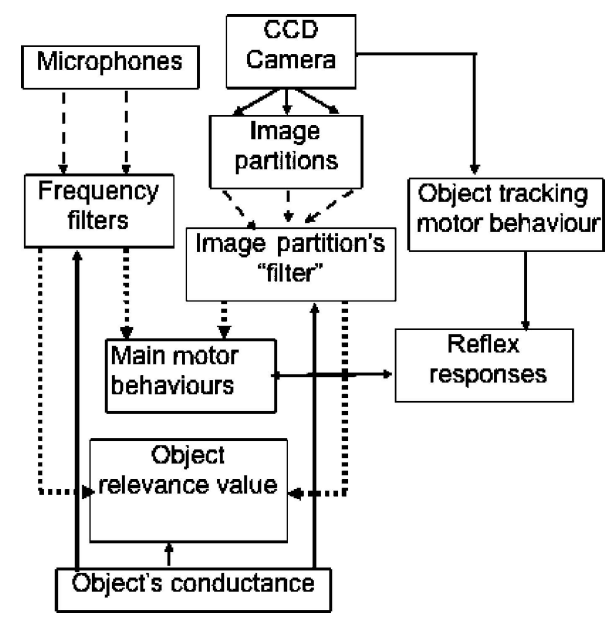

Figure $5 \mathrm{My}$ reading of the modular organization in Darwin VII robot.

The world signal relevance assessing is analogous to the ascending neuromodulatory value system (Schultz et al. 1997; Sporns et al. 2000).

The modules of pre-motor decision are analogous to the motor cortex and colliculus (Brooks 2002).

The set of modules of motor behavior correspond mainly to the spinal chord and lower brainstem.

The planning-and-sequencing modules of motor behavior correspond to the higher brainstem.

\section{OUR ROBOT BRAIN SCHEME IN OTHER IMPLEMENTATIONS}

\section{Darwin VII}

Figure 5 shows my reading of Darwin VII (Kirchmar and Edelman 2002) modularity. The two motor main areas (one reaching objects and one rejecting objects) are concentrated in a single box labeled main-motor behaviors. These behaviors are triggered depending upon the sound emitting characteristics of the surrounding objects, the object conductance and of a combination of image partitions rendered by an image-partition "filter". But in the case of the object-tracking motor behavior module the action is taken by the position of some recognized objects. The boxes are networks of neurons learning through plasticity (dotted arrows in the figure). Some plasticity networks are modulated by the influence of an object-relevance value module (double line dotted arrows). Continuous non-dotted arrows point to built-in connections.

\section{The brain scheme in D-VII}

Figure 6 shows the same basic robot brain scheme of the one in Figure 5, except that in D-VII robot the builders (Kirchmar and Edelman 2002) implemented three input signal modalities in the world signal processing set : sound image and conductance. The two motor areas in D-VII have been included into the set of modules of pre-motor decisions. The object tracking motor behavior is one of the 


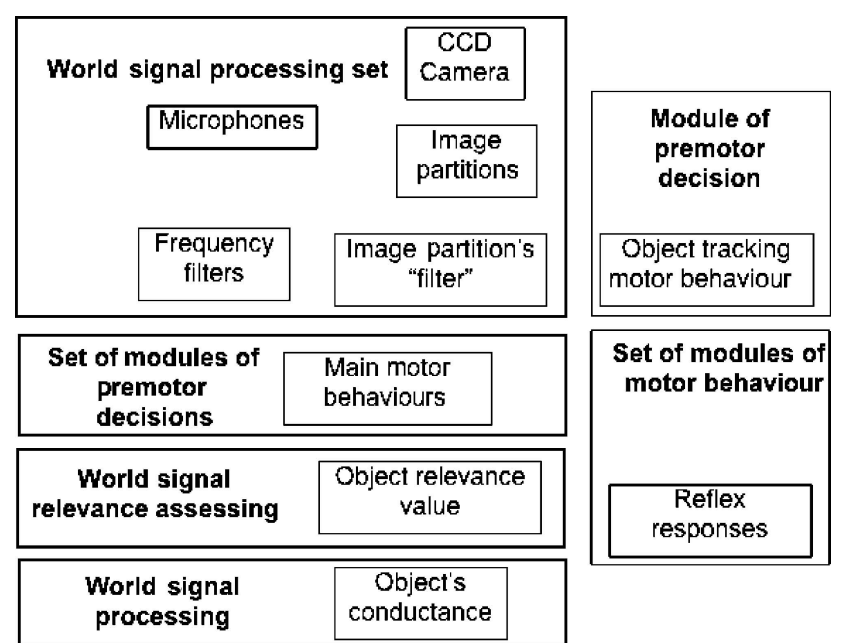

Figure 6 Darwin VII mapped into our robot brain scheme.

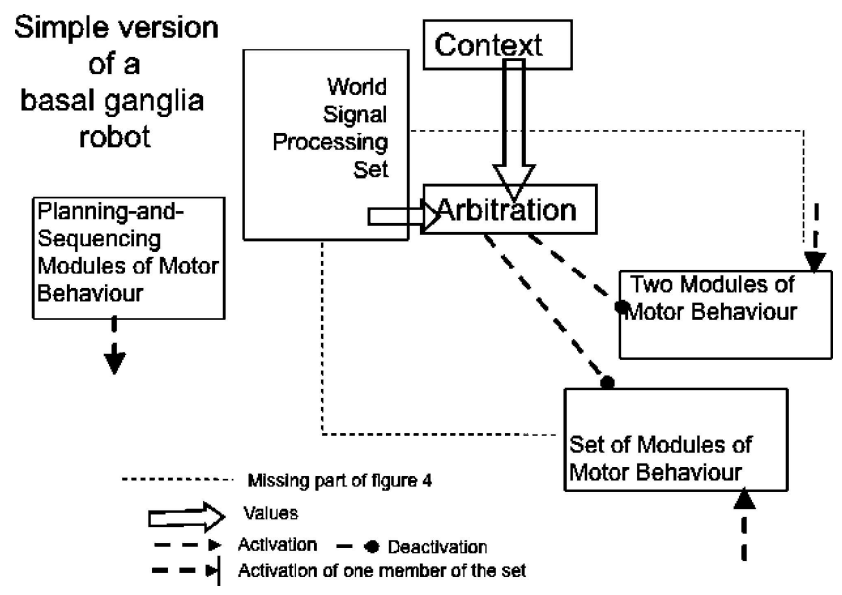

Figure 7 A basal gangla robot brain scheme mapped into our scheme.

pre-motor decision modules, and the object relevance value module corresponds to the class World Signal relevance assessing. Our robot is not for the moment a learning robot as D-VII is, but both robots have the same brain scheme.

\section{Basal ganglia robot}

Montes-Gonzalez and Negrete-Martinez (2006) have implemented a robot centered in the notion of the basalgangliaís behavior arbitration found in vertebrates. An ad hoc version compatible with our present scheme is depicted in Figure 7. Two new modules are added to the scheme of Figure 4, namely the context module and the arbitration module. The context module carries internal state information of the robot and sends it to the arbitration module. The arbitration module also receives information from the world signal processing set. The activation of all the modules of motor behavior (the selected modules and the rest) is on. The functionality of the arbitration module is to allow the 'salient' behavior of the two behaviors to be executed.

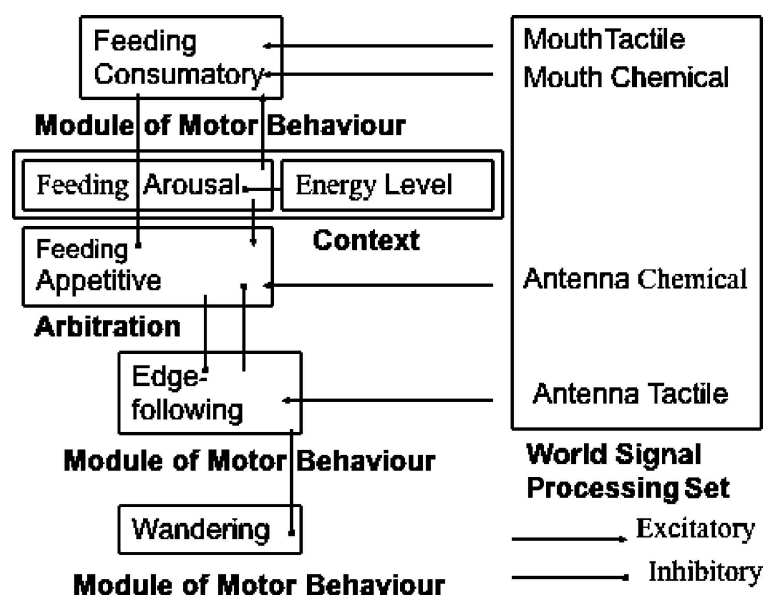

Figure 8 P. computatrix brain classified as in our scheme.

\section{P. computatrix}

Beer (1990) has implemented an insect-like robot called $P$. computatrix. In this robot, a set of six pace maker neurons (PM) or bursting neurons (one per leg) are interconnected through reciprocally inhibiting synapses in such a way that they generate a bursting pattern of activity of a tripod nature. Each PM controls the extensor and flexor movements of each leg. Alternating movements of the leg can be produced due to the control generated through backward and forward leg angle sensors (Pearson 1976). I classify this complex neural network as a module of motor behavior (see wandering behavior in Figure 8), similar to the wheels-rotation micro of our robot plus its motors. It is interesting to point out that there is another module of motor behavior (edge-following behavior in Figure 8) that mechanically perturbs the final motion process acting on the lateral extensor of the front legs, when there is an antennae tactile signal. The third and last module of motor behavior is the one concerned with the mouth movements (feeding consummatory in Figure 8). This module produces its behavior if the mouth is touching something with a chemical specificity, provided there is an internal stimulus coming from a context module (see Figure 8). The behavioral module feeding-appetitive in $P$. computatrix I classify it as an arbitration module since through information coming from chemicals in the antennae and from the context module the feeding appetitive module inhibits the edge-following and consequently the wandering. This facilitates the orientation of the insect toward the chemical (see Figure 8). All the sensorial inputs in the roach are taken as the world signal processing set.

\section{DISCUSSION}

A Robotic Brain Scheme must allow the implementation of many modules within the sub-schemes (D-VII). The robots must evolve by such modular additions (our Robot). New behaviors can be achieved within the scheme not only 
by module additions but by parameter modifications, as in our Robot: I can switch its behavior from Reaching toward light to avoiding it by simply changing the sign of one parameter in the set of pre-motors. Parameter modification is the case of the generation of new behaviors through unsupervised learning (D-VII).

Schemes should provide the necessary topology correspondence and the necessary functional correspondence with CNS as to provide experimental approach correspondence (all the robots compared here). The correspondence is at modular topology level (our robot and D-VII) but it is desirable also at sub modular level (D-VII and Basal Ganglia robot).

Schemes should provide basic principles of CNS organization as is the case of 'action induction' (NegreteMartinez 2006) (a result of modules' self-suppression in our robot) or the case of action selection by saliency evaluation in Basal Ganglia robot brain (Montes-Gonzalez and Negrete-Martinez 2006).

The case of a Module of Motion Behavior deactivation (Wandering) preceded by the mechanical effect of a Module of Motion Behavior (Edge-Following) in P. computatrix makes an interesting coupling of Motion behaviors.

Schemes should include Planning and Sequencing subschemes (ours) and arbitration sub-schemes (Basal Ganglion robot and $P$. computatrix).

\section{CONCLUSIONS}

A scheme of a robotic brain is useful because:

It provides a frame to discuss different robotic brain implementations (including invertebrates);

It suggests organizational ideas (insights) on the CNS that otherwise can be lost in the different implementations. Our scheme, for instance, stresses the importance of considering the necessary presence of:

Modules of motor behavior fed by pre-motor decision modules - the latter with a role of aiming-the-movement while the former with the role of developing the movement in detail;

Modules of planning and sequencing.

Our schemes:

a) suggest the necessary presence of arbitration modules.

b) provide clear CNS boundaries for the robot designer.

c) can be taken as bionic principles when tailored for engineering purposes.

\section{REFERENCES}

Negrete-Martínez J. 2006 Self-inhibiting modules can self-organize as a brain of a robot: A conjecture $\mathcal{7}$ App Bio Biomech, 3(1):23-27.

Schultz W, Dayan P, Montague PR. 1997. A neural substrate of prediction and reward. Science, 275:1593-1599.

Sporns O, Almassy N, Edelman GM. 2000. Plasticity in value systems and its role in adaptive behavior Adaptive Behav, 8:129-148

Brooks RA. 2002. Flesh and Machines, How Robots Will Change Us, New York: Pantheon Books, p. 65.

Kirchmar JL, Edelman GM. 2002. Machine psychology: Autonomous behavior, perceptual categorization and conditioning in a brain-based device, Cerebral Cortex, 12(8):818-830.

Beer RD. 1990. Intelligence as adaptive behavior: An experiment in computational neuroethology, Boston: Academic Press.

Pearson KG. 1976. The control of walking. Sci Amer, 235: 72-86.

Montes-González FMT, Prescott TJ, Negrete-Martínez J. 2006. Minimizing Human Intervention in the Development of the Basal Ganglia Inspired Robot Control. ISRA 2006 Proceedings. 

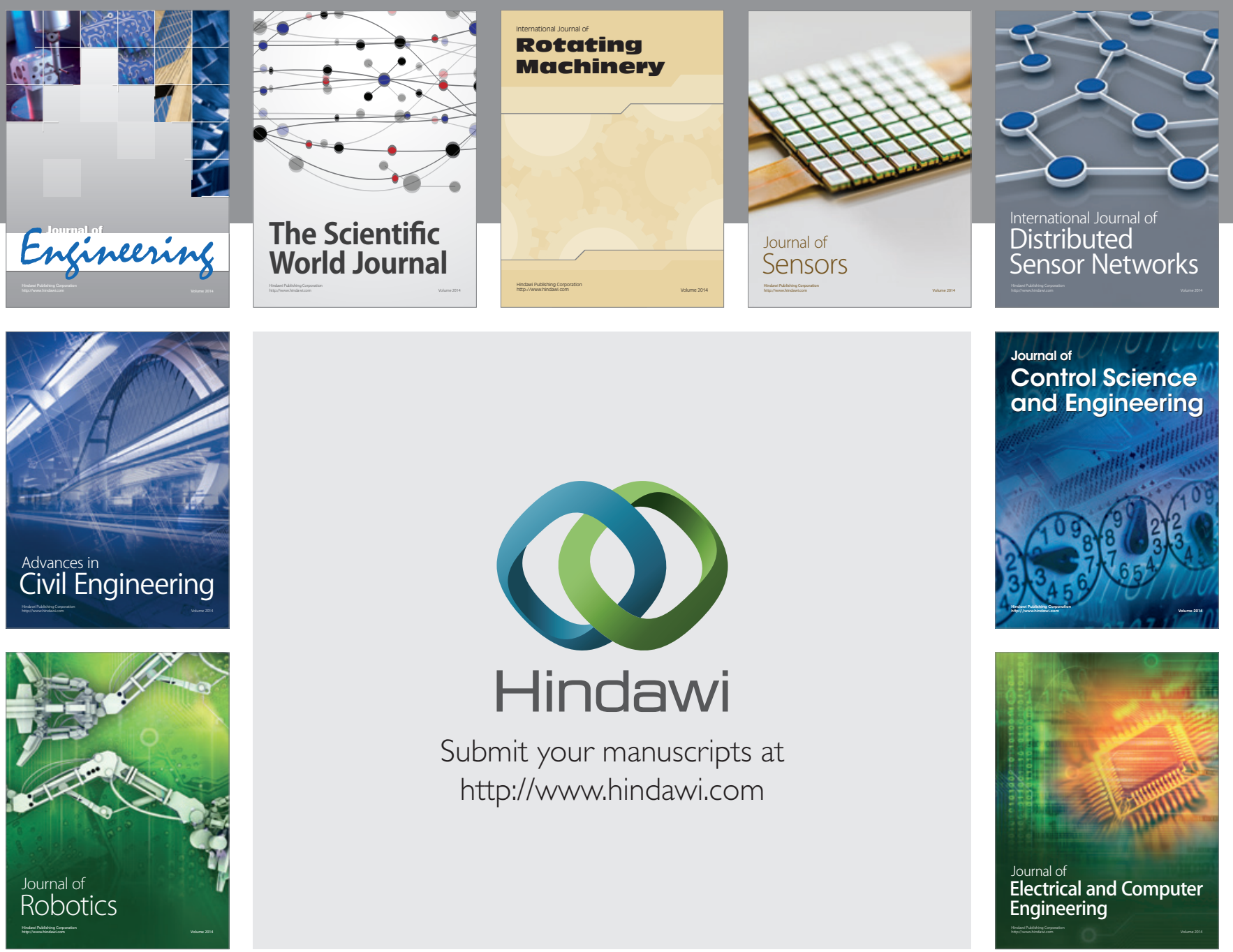

Submit your manuscripts at

http://www.hindawi.com
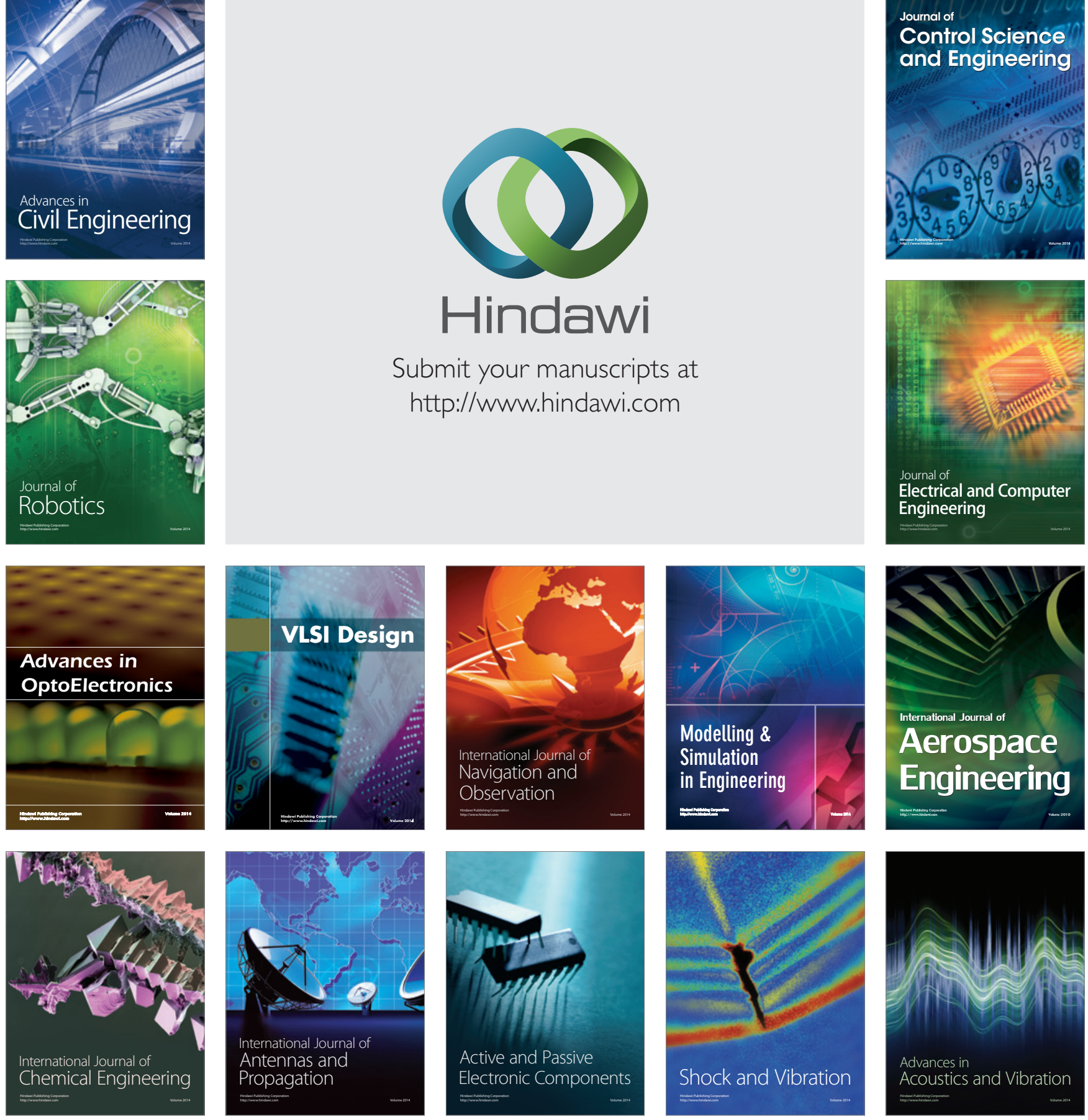